\section{$\underset{\substack{\text { hommes } \\ \text { \& migrations }}}{ }$}

\section{Hommes \& migrations}

Revue française de référence sur les dynamiques

migratoires

$1314 \mid 2016$

Migrations chinoises et générations

\title{
Arts, cuisine et design
}

La génération 1.75 de la diaspora chinoise à Milan et la création d'entreprises transnationales

\section{Giuseppina Merchionne et Hong Liu}

Traducteur : Catherine Guilyardi

\section{(2) OpenEdition}

\section{Journals}

Édition électronique

URL : http://journals.openedition.org/hommesmigrations/3632

DOI : 10.4000/hommesmigrations.3632

ISSN : 2262-3353

Éditeur

Musée national de l'histoire de l'immigration

Édition imprimée

Date de publication : 1 avril 2016

Pagination : 45-51

ISBN : 978-2-919040-35-3

ISSN : 1142-852X

Référence électronique

Giuseppina Merchionne et Hong Liu, «Arts, cuisine et design », Hommes \& migrations [En ligne],

1314 | 2016, mis en ligne le 01 avril 2019, consulté le 01 mai 2019. URL : http://

journals.openedition.org/hommesmigrations/3632 ; DOI : 10.4000/hommesmigrations.3632 


\title{
ARTS, CUISINE ET DESIGN LA GÉNÉRATION 1.75 DE LA DIASPORA CHINOISE À MILAN ET LA CRÉATION D'ENTREPRISES TRANSNATIONALES
}

\author{
par GIUSEPPINA MERCHIONNE, professeure de langue et culture chinoise, \\ université catholique de Milan, et HONG LIU, professeur d'histoire, Nanyang Technological \\ University (Singapour).
}

\author{
Tirant profit de la popularité croissante du "made in Italy", \\ les jeunes issus de l'immigration chinoise à Milan sont parvenus \\ à structurer leurs entreprises afin d'être opérationnels à \\ la fois en Italie et en Chine, dans des secteurs traditionnellement \\ associés à l'économie italienne comme la mode ou la \\ restauration. Jouant de leur double capital ethnique et du besoin \\ croissant de la part des consommateurs d'associer \\ des produits à des territoires, ces entrepreneurs épousent \\ les codes de l'économie transnationale, entre local et global.
}

Une nouvelle génération d'entrepreneurs sinoitaliens, dont les affaires sont structurées autour de transactions transnationales et d'activités transfrontalières, est en train d'émerger. Leurs identités sociale et culturelle se caractérisent certes par un mélange sino-italien, mais la balance penche clairement vers l'Italie. Les cas examinés dans cet article ont été choisis parmi les activités les plus représentatives de ces jeunes Sino-Italiens, tels que la restauration et l'industrie du luxe. On retrouve aussi ces jeunes dans la mode, le tourisme, le théâtre. Ils ont été sélectionnés dans le but de fournir la preuve empirique d'une nouvelle forme d'entreprenariat transnational entre l'Italie et la Chine, impliquant l'échange de produits, de biens, de capitaux et de savoir-faire, au-delà des frontières nationales. Notons qu'il s'agit de cas de réussites parmi les entrepreneurs sino-italiens.

Une diaspora est définie, de façon large, comme étant une "communauté ethnique séparée par des frontières nationales". Le transnationalisme est "le processus par lequel les migrants créent et entretiennent des relations sociales entre des lieux multiples qui lient leurs sociétés d'origine et d'accueil $3^{\prime \prime}$. Montrant l'existence, dans l'entreprenariat transnational, d'une double intégration, des chercheurs ont mis en évidence la possibilité d'une migration transnationale "à l'intérieur d'espaces sociaux fluides en constant remodelage, grâce à l'intégration simultanée des migrants dans plus d'une sociétét". Cependant, 
Les passants, rue de Belleville, mai 2016.

@ Camille Millerand

la plupart des études existantes se concentrent sur la première génération de migrants et portent peu d'intérêt aux descendants qui, pris entre deux cultures, n'ont pas de positionnement bien identifié dans chacune des sociétés. C'est à ce croisement que notre article entend apporter une contribution au corpus des études sur la migration. La génération 1.75 fait référence, dans cet article, aux Chinois d'Italie nés en Italie, mais renvoyés en Chine par leurs parents pour être confiés à leurs grands-parents entre 0 et 5 ans $s^{5}$. Ils sont ensuite revenus en Italie pour retrouver leurs parents et entrer à l'école.

\section{L'implantation des Chinois et le business ethnique chinois en Italie}

La première vague d'immigration chinoise en Italie arrivée dans les années 1920 s'est installée dans le quartier de Canonica-Sarpi à Milan. En 1962, les Chinois de la ville pénètrent pour la première fois le marché de la restauration grâce à un groupe d'entrepreneurs qui ouvrent le premier restaurant chinois. Il ne s'agit pas, pour eux, de monter une

5. L'expression “génération 1.5" désigne les migrants arrivés avant l'âge adulte et qui se trouvent entre la "génération 1" et la "génération 2". Afin d'affiner encore les profils des enfants de migrants, deux autres expressions sont apparues, celles de génération 1.25, pour désigner les jeunes ayant migré peu avant l'âge adulte et donc proches de la "génération 1", et celle de génération 1.75 pour désigner les enfants arrivés très jeunes et donc plus proches de la "génération 2" (note des coordinatrices). 
affaire profitable, mais de créer un endroit pour que les Chinois se retrouvent et mangent leur gastronomie.

Les résidents chinois en Italie en situation régulière étaient 320 794, début 2015. C'est la quatrième communauté étrangère après les Roumains, les Albanais et les Marocains ${ }^{6}$. À Milan, ils sont plus de 22000 (en 2012), c'est-à-dire plus de $9 \%$ des résidents étrangers, et les moins de 35 ans représentent près de $60 \%$ des résidents chinois de la ville?

En 2014, 69410 entreprises en exercice sont tenues par des Chinois, ce qui représente une croissance de 39,2 \% depuis 2009. En 2015, les Chinois possédaient 42705 entreprises $^{8}$, soit 11,3\% du total des entreprises étrangères. Parmi ces entreprises, 15252 ont leur siège social en Lombardie, suivie de la Toscane (12 310) et de la Vénétie (8 367), selon le rapport de la Chambre de commerce de Milan de 2015.

Les entreprises au capital exclusivement chinois à Milan sont concentrées dans trois secteurs : le commerce (1 089 sociétés, 36,3\% du total), les restaurants, bars et hôtels $(834,27,8$ \%) et diverses activités de services (505, 16,8 \%). Malgré la crise économique de 2008-2009, l'esprit entrepreneurial n'a pas reculé : le nombre total d'entreprises chinoises à Milan a augmenté de $12 \%$ entre 2010 et $2012^{9}$.

Certaines grandes entreprises ont su saisir des opportunités en ouvrant des joint-ventures avec des compagnies d'import-export. Leur chiffre d'affaires représente aujourd'hui des dizaines de millions d'euros et elles sont devenues des entreprises multinationales.

\section{La génération 1.75}

Les spécialistes de la migration ont étudié la deuxième génération et leur changement d'identité dans la société d'accueil, mais définir cette deuxièmegénération est moins évident qu'il n'y paraît. Cette catégorie conceptuelle recouvre des cas très différents, depuis les enfants nés et élevés dans la société d'accueil aux adolescents retrouvant leurs parents après avoir déjà vécu un processus de socialisation dans le pays d'origine ${ }^{10}$. Une étude récente affirme que le concept de "deuxième génération de migrants" pose problème et suggère de les appeler "première génération d'Italiens", un concept plus dynamique et tourné vers l'avenir ${ }^{11}$. Leur contribution à la société italienne a été reconnue: "Les migrants de la Les spécialistes de la migration ont étudié la deuxième génération et leur changement d'identité dans la société d'accueil, mais définir cette seconde génération est moins évident qu'il n’y paraît.

deuxième génération peuvent enrichir la société italienne de multiples façons, et ils le font. Une majorité parle deux ou trois langues couramment, ce qui est un avantage et une avance certaine dans un pays où la maîtrise des langues étrangères est traditionnellement pauvre ${ }^{12}$."

Rumbaud a classé les différentes générations de migrants selon leur niveau d'éducation dans le pays d'accueil, créant des variantes entre "génération 1.5, 1.25 et $1.75^{13}$ ". La génération 1.5 commence son processus de socialisation à l'école primaire dans le pays d'origine, mais termine sa scolarité dans le pays d'accueil. La génération 1.25 migre entre 13 et

6. Antonella Ceccagno, "Compressing diversity: ethnicization of the workforce and outsourcing of social reproduction as assets for the italian fashion industry", in Journal of Population and Social Studies, vol. 2, n 1, 2016, pp. $92-111$; Fondazione Ismu, Ventunesimo Rapporto sulle migrazioni 2015 (21 Rapport sur les migrations, 2015). 7. Statistiques de la ville de Milan (Comune di Milano), Rapport sur l'entreprenariat individuel 2013, 2012 et 2011. 8. Antonella Ceccagno, op. cit. 9. Chambre de commerce de Milan (Camera di Commercio di Milano), Rapport sur les registres de sociétés : données sur les entrepreneurs individuels aux troisièmes trimestres, 2013. 10. Maurizio Ambrosini, Il futuro in mezzo a noi. Le seconde generazioni scaturite dall'immigrazione nella società italiana dei prossimi anni (Le futur parmi nous. La seconde génération, résultat de l'immigration dans la société italienne dans les années à venir), in Maurizio Ambrosini, Stefano Molina (dir.), Seconde generazioni. Un'introduzione al futuro dell'immigrazione in Italia (La seconde génération. Une introduction à l'immigration à venir en Italie), Torino, Edizioni Fondazione Giovanni Agnelli, 2004. 11. Bjørn Thomassen, "Second generation immigrants" or "Italians with immigrant parents" ? Italian and european perspectives on immigrants and their children", in Bulletin of Italian Politics, vol. 2, n 1, 2012, pp. 21-44. 12. Ibid. 13. Rubén G. Rumbaut, "Ties that bind: immigration and immigrant families", in Alan Booth, Ann C. Crouter, Nancy S. Landale (dir.), Immigration and The Family: Research and Policy on U.S. Immigrants, Mahwah, NJ, C. Lawrence Erlbaum Associates, 1997. 
17 ans. La génération 1.75 migre avant le début de la scolarisation obligatoire ${ }^{14}$. Quelles sont les différentes générations d'entrepreneurs chinois à Milan? Peut-on tous les appeler huashāng (commerçants de la diaspora chinoise) ? À quel degré reproduisentils l'organisation sociale et économique typique de leurs prédécesseurs ? Ont-ils développé, comme leurs parents, la huashang wenhua (la culture commerciale chinoise) attribuée

Le processus d'intégration

fait appel à d'autres besoins dont l'acquisition de valeurs culturelles modernes à l'école,

dans leur environnement social et culturel et professionnel, soit en dehors de la communauté. aux Chinois de la diaspora ${ }^{15}$ ? Les communautés chinoises de différents pays, dont l'Italie, donnent la priorité à la permanence de la langue et à l'éducation, afin que les jeunes générations, les huayi (descendants de Chinois), n'oublient pas leurs racines. Mais les jeunes générations ne suivent pas, en général, les mêmes modèles migratoires que leurs aînés. Ainsi, la langue chinoise n'est pas considérée par eux comme essentielle pour leur éducation ni pour leur reconnaissance sociale. Le processus d'intégration fait appel à d'autres besoins, dont l'acquisition de valeurs culturelles modernes à l'école, dans leur environnement social, culturel et professionnel, soit en dehors de la communauté. Ils favorisent aussi la maîtrise d'autres langues au détriment du chinois.

\section{Négocier avec les marqueurs identitaires}

Parmi les huayi, dont $30 \%$ sont nés en Italie ${ }^{16}$, l'acquisition d'éléments structurels non chinois n'est pas une menace pour la permanence de la culture d'origine. Si les Chinois des deuxième et troisième générations ne sont pas véritablement scolarisés dans le pays d'origine, il est peu probable qu'ils aient une idée de ce qui diffère entre la "culture d'origine" et leur vie quotidienne dans la diaspora, d'autant moins si la génération qui les a précédés n'a rien transmis. Ces jeunes gens sont souvent "trop occidentalisés" pour se sentir à l'aise en tant que Chinois, mais encore "trop Chinois" pour accepter que le fait d'être Chinois soit un handi$\operatorname{cap}^{17}$. Il est important de comprendre en quoi la permanence ou la perte de leur "sinitude" a une incidence sur la réussite de leur entreprise, en la favorisant ou la freinant.

Les caractéristiques linguistiques de la diaspora chinoise en Lombardie illustrent ce que l'on observe dans le reste de l'Italie, en terme d'origine géographique et de dialectes parlés. À Milan comme à Rome, en dehors des Chinois de Zhejiang et, dans une moindre mesure, du Fujian, la communauté chinoise comprend une petite minorité de Taïwanais qui parlent le dialecte minnan (Fujian du Sud). Les recherches montrent que les différences de classes sociales et de situations économiques entre Chinois dépendent grandement de leur lieu d'origine : les Chinois du Zhejiang sont plutôt entrepreneurs, ceux du nord-est de la Chine sont plutôt salariés ${ }^{18}$. Ces jeunes gens sont en général trilingues (italien, chinois et dialecte) capables de naviguer dans différents contextes sociaux et familiaux $^{19}$. La question du choix de l'éducation des jeunes générations n'est pas un phénomène propre à la situation italienne. Dans les communautés chinoises plus anciennes en Asie du Sud-Est et en Amérique, le choix est facilité par la présence d'écoles suivant un cursus et des méthodes éducatives proches de ceux en place en Chine. Le rejet de la culture chinoise par les jeunes n'a rien de surprenant. C'est une attitude fréquente, surtout pendant l'adolescence, chez les jeunes de la diaspora qui considèrent la Chine comme un pays arriéré. Ils ressentent leur condition d'immigrés chinois comme un marqueur les séparant clairement de leurs pairs italiens, et ce malgré une mobilité sociale liée à leur réussite économique souvent meilleure que celle 
de la moyenne parmi les jeunes Italiens. Considérant que leur futur est en Italie, ils recherchent un niveau de vie identique à celui des Italiens afin de se hisser au-dessus du statut de "parias". Leur désir d'être "comme les autres" s'exprime aussi dans leur refus de parler la langue d'origine : nombreux sont les jeunes, même ceux qui ont étudié quelques années en Chine, qui refusent de s'exprimer autrement qu'en italien, même en présence de leurs parents. Grâce à leur éducation, ils sont totalement intégrés dans la société italienne mais sont conscients d'avoir un autre atout, celui de connaître la langue et la culture de deux pays, ce qui leur permet de travailler non seulement pour des entreprises italiennes, mais aussi de faire des affaires entre la Chine et l'Italie ${ }^{20}$.

Pour ces jeunes gens, devenir entrepreneur reste une décision prise dans le noyau familial. De nos jours comme autrefois, la pérennité entrepreneuriale est le résultat d'un effort collectif des membres de la famille dans le management et la gestion de la société. Cela explique pourquoi nombre d'entre eux entrent dans des universités, italiennes ou étrangères, pour suivre des cursus en sciences économiques, business, management, droit, design ou génie mathématique ou informatique. Aujourd'hui, ces jeunes entrepreneurs ne s'affairent plus à démontrer leur succès, mais plutôt à pérenniser et à faire prospérer leur affaire grâce à une planification économique prudente et une bonne gestion des ressources.

\section{L'imbrication du transnational et du local}

Le "made in Italy" est comme un label reconnu mondialement évoquant des produits et un style de vie typiquement italiens, touchant particulièrement la gastronomie, l'ameublement intérieur, la mode vestimentaire et les industries mécanique et automobile. Depuis quelques dizaines d'années, deux catégories de produits se sont imposés sur le marché global de façon simultanée : le "made in Italy", venu du monde occidental, et son pendant oriental, le "made in China". Le premier est synonyme de produits de luxe réservés à une clientèle choisie et très fortunée, le second, jusqu'à récemment, est synonyme de produits manufacturés visant un large panel de
Eline et Celine Liu, en charge de l'entreprise Abilandia, import de carrelage de style italien fabriqué en Chine. (C) G. Merchionne

consommateurs, bien moins aristocratiques. Ces labels illustrent deux réalités différentes du marché et des habitudes de consommation, sur une route allant de l'Italie à la Chine et vice versa.

Nous nous intéressons ici aux entrepreneurs actifs dans différentes industries et services liés au besoin d'un territoire, qui adoptent une attitude différente dans leur mode de vie, leur travail et la gestion de leur entreprise que celle de leurs parents et de leurs homologues italiens. Un premier moment clé de changement dans les choix d'activité, en répercussion de la crise du syndrome 
respiratoire aigu sévère (SRAS) en Chine et en Asie du Sud-Est (2003), a consisté dans la reconversion de la plupart des restaurants chinois en restaurants japonais, voire italiens. Plus tard, la création d'entreprises dans de nouveaux secteurs d'activités, tels le high-tech, le design, la mode, la finance, dans un contexte de crise économique européenne, a poussé la deuxième génération de Chinois à explorer d'autres stratégies entrepreneuriales plus adaptées aux changements.

Bien que les jeunes Sino-Italiens investissent de nouveaux secteurs et développent de nouvelles stratégies d'entreprise, ils recourent encore souvent à des moyens de financement traditionnels : soit indirectement grâce à des fonds alimentés par des membres de la famille ou des compatriotes en Italie, en Chine ou dans d'autres pays européens, soit directement par des prêts réservés au startup ou grâce aux cérémonies familiales (mariages) pendant lesquelles des sommes importantes sont échangées, selon la logique de la réciprocité.

En Italie, ils présentent leur entreprise comme des partenaires de compagnies italiennes, alors qu'en Chine ils sont considérés comme les intermédiaires les plus appropriés pour les entreprises chinoises qui voudraient investir en Italie ${ }^{21}$. Parmi les nouvelles stratégies, les cas suivants illustrent celle qui consiste à réinterpréter le "made in Italy".

\section{L'empire du vin}

Luigi Wu, né à Wenzhou en 1974, est arrivé en Italie à 14 ans et étudie la cuisine en lycée professionnel. En 2010, il est admis par l'association des sommeliers italiens, l'AIS. Il commence alors à exporter du vin italien en Chine et ouvre sa propre société à Shanghai en 2011. La même année, il obtient le titre de sommelier de la World Sommelier Association et il est reconnu comme le premier sommelier chinois en Italie. Sa société est domiciliée à Brescia, mais les entrepôts sont dans la zone franche de Shanghai Yangshan. Sa compagnie d'import-export s'appelle Yidi Wine. Yi est le caractère chinois pour Italie, et Di pour empire, autrement dit l'Italie est l'empire du vin, où il possède un vignoble qui produit du vin exclusivement pour sa marque.

\section{Le restaurant Italien d'un entrepreneur chinois}

Né à Wencheng en 1981, Francesco Wu est arrivé à Milan à 8 ans pour rejoindre son père. En 2005, il est diplômé en génie électronique de Politecnico di Milano, mais décide d'acheter un restaurant dans la banlieue de Milan, qui marie la tradition culinaire méditerranéenne et le management chinois. Il est aujourd'hui troisième dans le classement des 70 meilleurs restaurants de Milan.

En 2007, il intègre Associna, l'association des entrepreneurs chinois de la deuxième génération, créée deux ans plus tôt. Il devient président de sa branche lombarde. Sa popularité grandit grâce à sa médiatisation dans des émissions télévisées et dans la presse italienne. En 2012, il créé l'Union des entrepreneurs sino-italiens (UNIIC), qui réunit 300 membres, en majorité des Chinois. Sa vie et son expérience ont été racontées dans un célèbre magazine italien, Espresso, et dans l'édition européenne du China Daily, en octobre 2013.

\section{Le transfert de technologie du "made in Italy"}

Les sœurs Eline et Celine ont suivi les mêmes études en commerce et langues étrangères à l'université catholique. Elles n'ont jamais parlé chinois, mais le comprennent, et ont des fiancés et petits amis italiens. Eline Liu est née à Paris en 1988. Elle s'est installée à Milan avec sa famille à lâge de 2 ans. Son père a ouvert, en 1995, à Milan, Liu Liu's World Import Export, spécialisé dans l'exportation de design, de machinerie et de matières premières pour la production de tuiles d'Italie en Chine et 
l'importation du produit fini de Chine en Italie. La forte hausse de l'activité a poussé la famille à lancer une autre entreprise appelée Abilandia (Abi : vivre plus longtemps et land: terre), en 2006, dont la jeune Eline est devenue propriétaire, dans le but de faire le lien avec le marché italien. La production en Chine est orchestrée par la mère, qui s'est réinstallée à Foshan de façon permanente. Celine est aussi née à Paris juste avant leur déménagement. Elle travaille pour Abilandia tout en poursuivant ses études, avec pour mission de développer la vente de vin italien comme activité annexe (auprès des fournisseurs de tuiles italiennes de Chine pour qu'ils s'en servent de cadeaux promotionnels).

\section{Conclusion}

Cette étude montre qu'un nouveau type d'entreprises ethniques chinoises a émergé et s'est développé en Italie, non seulement dans le secteur du luxe et le marché du "made in Italy", mais aussi dans l'immobilier et le tourisme. De jeunes guides chinois conduisent une foule de touristes de leur pays d'origine sur les lieux de la mode mais aussi sur le marché de l'immobilier, où un grand nombre cherche à investir dans des propriétés privées ou des immeubles. Ils viennent davantage acheter des biens de luxe que visiter les musées, monuments et sites archéologiques, autrefois les vrais symboles du "made in Italy".

Ces entreprises font preuve d'un double processus d'intégration du fait que les nouveaux entrepreneurs de la génération 1.75 ont su utiliser leur double capital ethnique, les affinités culturelles en Italie et Chine, et que les entreprises sont installées, physiquement et financièrement, dans les deux pays. C'est un schéma que l'on retrouve parmi les nouveaux migrants chinois de Singapour. Il est démontré que, par comparaison, l'intégration dans un seul pays est plus simple que l'intégration duale ou transnationale, qui dépend des institutions sociales des deux sociétés et des capacités transnationales du professionnel. Étant des immigrés transnationaux, les entrepreneurs chinois de Singapour s'investissent dans des activités économiques plus globales que s'ils étaient dans des sphères distinctes et séparées. Leurs pratiques professionnelles sont menées et régulées par les sociétés dans lesquelles ils agissent, plutôt que dans un vide social. Le pays d'origine et le pays d'accueil deviennent leurs deux principaux terrains d'activités sociales et économiques. En d'autres mots, les pratiques transnationales et l'intégration locale ont été intégrées dans un même processus, et sont des stratégies complémentaires renforçant les efforts des immigrés pour cumuler un capital social et économique dans le pays d'origine et le pays d'accueil ${ }^{22}$.

Dans l'espace de cet article, nous ne pouvons pas parler d'autres facteurs importants pour expliquer les caractéristiques des nouveaux entrepreneurs transnationaux chinois de Milan, tels que l'économie politique des espaces urbains et la dynamique de la restructuration urbaine ${ }^{23}$, qui ont un effet direct sur les activités des Chinois de Milan, de la restauration à l'industrie de la mode. Par ailleurs, le développement rapide des activités du marché ethnique des Chinois de Milan a été facilité par l'expansion des investissements directs à l'étranger (IDE) des Chinois en Italie qui ont visé "l'acquisition de savoir-faire technologiques et de design et su tirer profit de compétences locales, dans des domaines spécialisés de l'industrie automobile et des appareils électroménagers ${ }^{24 "}$. Ces ajustements institutionnels nationaux et cet environnement mondialisé, dont fait partie la croissance de la Chine, sont le cadre qu'il faut prendre en compte pour une interprétation compréhensive de l'entreprenariat chinois en Italie.

Texte traduit de l'anglais par Catherine Guilyardi. 